
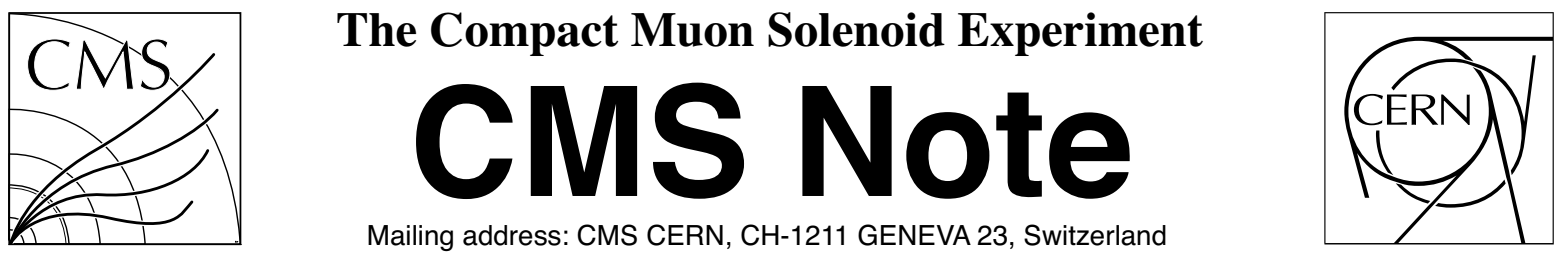

11 November 2020 (v2, 22 February 2021)

\title{
Influence of Surface Damage and Bulk Defects on the Interstrip Isolation of p-type Silicon Strip Sensors
}

\author{
Jan-Ole Müller-Gosewisch, Alexander Dierlamm, Andreas Nürnberg
}

\begin{abstract}
Silicon strip sensors of upcoming tracking detectors in high luminosity colliders usually consist of a p-doped bulk with n-type strip implants. The general consensus is that such a design requires an additional interstrip isolation structure such as a p-stop implant. If there is no additional implant between the strips, it is expected that the strip isolation will be insufficient. Before irradiation, impurities and defects in the material lead to positive charge in the oxide and $\mathrm{Si} / \mathrm{SiO}_{2}$ interface which attracts electrons from the bulk. Those electrons accumulate just beneath the surface and between the $\mathrm{n}^{+}$strip or pixel implants, which decreases the interstrip resistance significantly. Ionising radiation introduces even more charge inside the silicon dioxide, which further decreases the interstrip resistance. Contrary to that expectation of a decreasing interstrip resistance due to irradiation, a high interstrip resistance was sometimes observed after proton irradiation. Hence, bulk defects induced by proton irradiation seem to have a non-negligible impact on the strip isolation. Therefore, an irradiation campaign with sensors without any interstrip isolation implant has been performed. The use of sensors without isolation structures provides deeper insight into the dominant isolation effects. The sensors' performance is first evaluated before irradiation. Afterwards, a set of sensors is irradiated with different particles in order to systematically introduce a mixture of surface and bulk defects. This enables the distinction and leads to a better understanding of the different effects. Finally, a combined TCAD model is derived which is able to describe the beneficial effect of bulk defects and the complicated interplay between surface and bulk damage.
\end{abstract}




\title{
Influence of Surface Damage and Bulk Defects on the Interstrip Isolation of p-type Silicon Strip Sensors
}

\author{
Jan-Ole Müller-Gosewisch, Alexander Dierlamm, Andreas Nürnberg \\ Karlsruhe Institute of Technology \\ Hermann-von-Helmholtz-Platz 1 \\ Eggenstein-Leopoldshafen, Germany \\ E-mail: jan-ole.gosewisch@kit.edu
}

\begin{abstract}
AвSTRACT: Silicon strip sensors of upcoming tracking detectors in high luminosity colliders usually consist of a p-doped bulk with n-type strip implants. The general consensus is that such a design requires an additional interstrip isolation structure such as a p-stop implant. If there is no additional implant between the strips, it is expected that the strip isolation will be insufficient. Before irradiation, impurities and defects in the material lead to positive charge in the oxide and $\mathrm{Si} / \mathrm{SiO}_{2}$ interface which attracts electrons from the bulk. Those electrons accumulate just beneath the surface and between the $\mathrm{n}^{+}$strip or pixel implants, which decreases the interstrip resistance significantly. Ionising radiation introduces even more charge inside the silicon dioxide, which further decreases the interstrip resistance. Contrary to that expectation of a decreasing interstrip resistance due to irradiation, a high interstrip resistance was sometimes observed after proton irradiation. Hence, bulk defects induced by proton irradiation seem to have a non-negligible impact on the strip isolation. Therefore, an irradiation campaign with sensors without any interstrip isolation implant has been performed. The use of sensors without isolation structures provides deeper insight into the dominant isolation effects. The sensors' performance is first evaluated before irradiation. Afterwards, a set of sensors is irradiated with different particles in order to systematically introduce a mixture of surface and bulk defects. This enables the distinction and leads to a better understanding of the different effects. Finally, a combined TCAD model is derived which is able to describe the beneficial effect of bulk defects and the complicated interplay between surface and bulk damage.
\end{abstract}

KEYwORDS: Radiation damage to detector materials (solid state), interstrip isolation and resistance, surface and bulk damage, tracking detectors 


\section{Contents}

1 Introduction 1

2 Measurement and Simulation Procedure 2

$\begin{array}{lll}2.1 & \text { Measurement Procedure } & 2\end{array}$

2.2 Device Simulation $\quad 2$

3 Investigations pre Irradiation $\quad 3$

3.1 Interstrip Resistance pre Irradiation $\quad 3$

3.2 Isolation Mechanism and Impact of the Doping Concentration 4

4 Interstrip Resistance with pure Surface Damage $\quad 7$

$\begin{array}{lll}4.1 & \text { Experimental Results } & 7\end{array}$

$\begin{array}{lll}4.2 & \text { Surface Defect Simulations } & 7\end{array}$

5 Interplay of Surface and Bulk Defects 9

5.1 Irradiation Procedures 9

$\begin{array}{ll}5.2 & \text { Experimental Results } \\ 5.3\end{array}$

$\begin{array}{lll}\text { 5.3 Simulated Impact of Bulk Defects on the Interstrip Isolation } & 11\end{array}$

$\begin{array}{lll}5.4 & \text { Comparison of Simulations to Experimental Results } & 14\end{array}$

$\begin{array}{lll}6 & \text { Conclusion } & 16\end{array}$

\section{Introduction}

The majority of silicon sensors for future tracking detectors will be made on p-type bulk material with n-type readout implants. Due to the presence of positive oxide charge inside the $\mathrm{SiO}_{2}$ surface of the device, an electron layer is formed directly below the $\mathrm{Si} / \mathrm{SiO}_{2}$ interface in the bulk. This electron channel short-circuits the pixels or strips and consequently the spatial resolution decreases. To prevent this, an additional interstrip isolation implant is usually added. Commonly used structures are a p-stop implant or a p-spray layer or a combination of both. This implementation guarantees the operability of the sensor and is used in tracking detectors at high luminosity collider experiments [1].

However, in previous studies an unexpectedly high interstrip isolation was also observed for low p-stop doping concentrations or even without using any isolation implant, especially after irradiation with protons which deposit a high ionising dose in the surface of the device [2]. Since protons additionally induce bulk damage, the hypothesis is that the bulk defects help to establish a strip isolation.

The aim of the following study is to gain a better understanding of the interplay of surface defects and bulk defects. Capacitively coupled $\mathrm{n}^{+}$-in-p type strip sensors $(90 \mu \mathrm{m}$ strip pitch and 
$1.45 \mathrm{~cm}$ strip length) without any interstrip isolation structures are investigated. These miniature strip sensors are part of the prototype sensor wafers for the CMS Phase-2 Outer Tracker R\&D program [3] for the High Luminosity LHC (HL-LHC). Hence, the material is the same as the one which will be used in the new CMS tracker. The difference is solely that the design of these sensors does not feature a p-stop implant between adjacent strips which would disguise the effects studied here. Hence, if not otherwise specified sensors without any isolation structures are discussed.

The measurement and simulation procedures are described in Section 2. Pre-irradiation behaviour and the influence of the doping concentration on the strip isolation is investigated in Section 3. Afterwards, the impact of pure surface damage on the interstrip isolation is evaluated. In order to understand the dominant effects regarding the interstrip isolation, a TCAD simulation model has been adapted which describes the interstrip resistance measurements accurately after $\mathrm{X}$-ray irradiation. This model and a comparison with respective measurements is presented in Section 4. The impact of bulk damage applied by irradiation with neutrons and protons is described in Section 5 and compared to a composite TCAD model which reproduces the measurements when bulk and surface damage are present at the same time.

\section{Measurement and Simulation Procedure}

\subsection{Measurement Procedure}

When operated as particle detectors, the strip sensors are biased under reverse bias by applying a negative potential on the backside while grounding the strip implants via a bias ring. Two adjacent DC pads are contacted with two probe needles as illustrated in Figure 1a in order to measure the interstrip resistance. A bias potential ramp of up to $10 \mathrm{~V}$ is applied on one needle while the current flow through the second needle is measured. The current is a composition of the strip leakage current of the substrate and the current flow between the two strips. Due to the low potential ramp, the strip current is increasing in dependence of the voltage, as indicated in Figure 1b. The value of the interstrip resistance is obtained by the inverse slope of a linear fit of the strip current over voltage curve. With this procedure the strip leakage current, which would be the offset of the linear fit, is separated from the interstrip current. Before irradiation and after X-ray irradiation, the interstrip voltage ramp is performed up to only $1 \mathrm{~V}$ to prevent altering the electric field distribution near the strips. However, the bulk leakage current in reverse bias is increased by several orders of magnitude after proton and neutron irradiations. Hence, an interstrip voltage ramp to $10 \mathrm{~V}$ is used to reliably determine the interstrip resistance and to cope with the measurement noise [4] added by the increased leakage current in irradiated sensors.

\subsection{Device Simulation}

A two-dimensional representation of the device is implemented in the finite-element simulation framework Synopsys Sentaurus TCAD [5]. Figure 2a shows the doping levels of the simulated device. To reduce the computational effort, only two half strips are implemented. Boundaries of the device are treated with Neumann conditions so that current can exclusively flow through the metal contacts. The highly n-doped strip implants are coloured in red and the p-doped bulk in blue. On top of the strip implants there is an AC coupled aluminium pad (with a $0.25 \mu \mathrm{m}$ thin coupling 


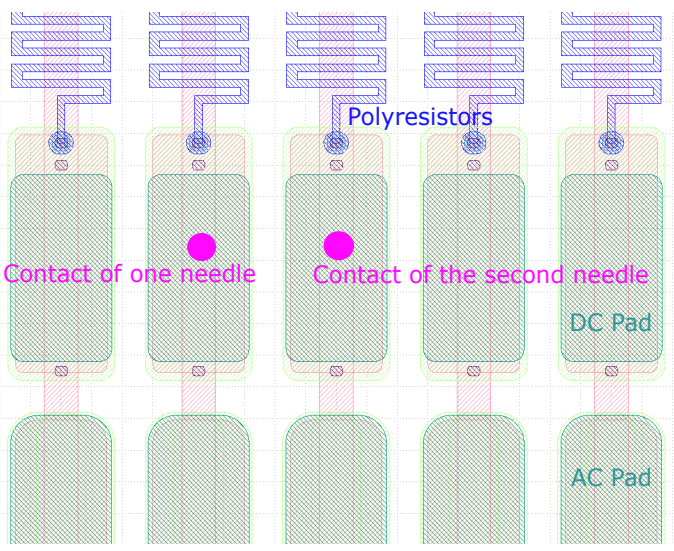

(a)

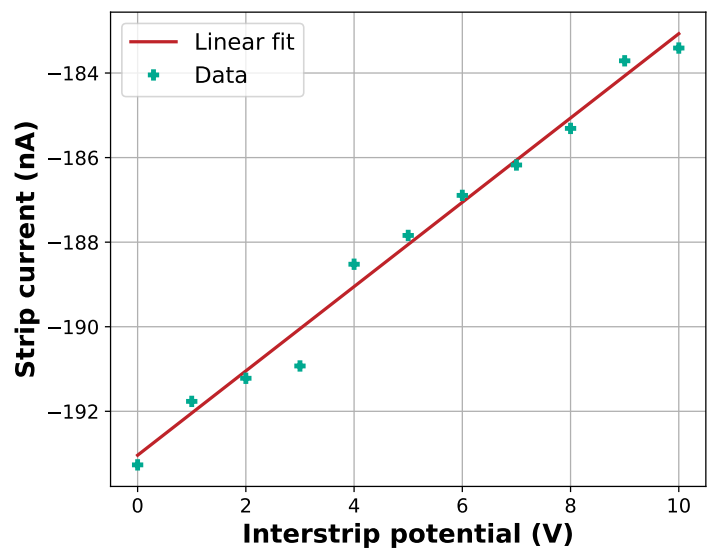

(b)

Figure 1: For the interstrip resistance determination two adjacent DC pads are contacted with two probe needles (a). A low voltage ramp is applied on one needle and the strip current flow is measured through the other needle for a sensor without interstrip implant and after irradiation with protons (b). The total current is negative due to the dominating bulk leakage current. Since the low voltage ramp on the neighbouring strip is positive, a positive current is added. The interstrip resistance can then be obtained by the inverse slope of this characteristic and is in this case $1 \mathrm{G} \Omega$.

oxide) and a larger metal overhang. A thicker surface oxide is placed between the two half-strips. Net charge inside the oxide is reflected by an effective fixed charge at the $\mathrm{Si} / \mathrm{SiO}_{2}$ interface. The simulated device properties are scaled with the strip length in order to describe the physical device using the symmetry along the strips. The thickness of the sensor bulk is always similar to the active thickness of the physical device, which varies from 200 to $290 \mu \mathrm{m}$ for different batches. A highly p-doped backside implant with an aluminium contact is implemented to apply the reverse bias. However, since the following studies focus on the surface region, these implants are not displayed here. In Figure $2 b$ the finite-element mesh in the surface region is shown. The mesh's point distance varies according to changes in the doping concentration. In the bulk region a mesh point distance of several micrometers is sufficient. Coming closer to the interface region the point distance is decreased to a few nanometers. In order to determine the interstrip resistance, a low voltage difference of $1 \mathrm{~V}$ is applied between the two strips and the current flow between the two strip contacts is extracted, similar to the measurement of the physical device.

\section{Investigations pre Irradiation}

\subsection{Interstrip Resistance pre Irradiation}

Before any irradiation, the interstrip resistance is measured at a controlled temperature of $+20^{\circ} \mathrm{C}$. A set of at least five strips per voltage setting was measured and the mean values with the associated standard deviations are indicated as datapoints with error bands in the following plots. The interstrip resistance of a sensor without any interstrip isolation implant and for comparison of another sensor with a p-stop implant are illustrated in Figure 3. The figure shows the dependence of the measured 


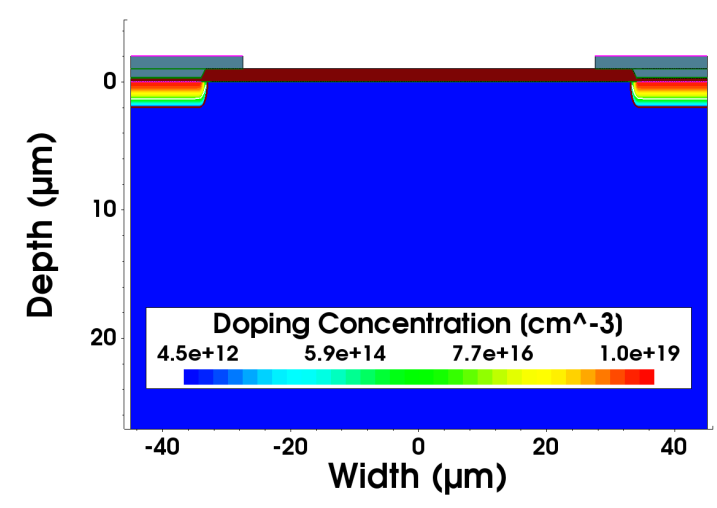

(a)

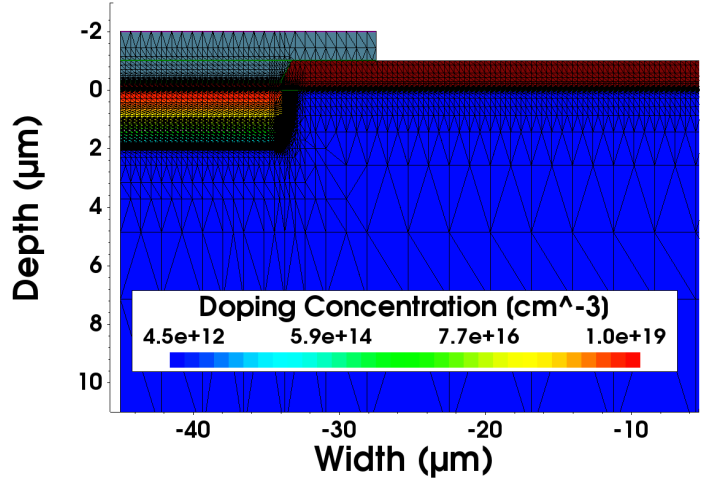

(b)

Figure 2: Top part of the simulated device with the most relevant structures (a) and with the finiteelement mesh for the simulation (b). The mesh is considerably more dense towards the surface and strip implants.

interstrip resistance on the applied reverse bias voltage. In order to give values independent of the strip length, the resistance is normalised to the strip length. Since the p-stop implant prevents the electron accumulation beneath the surface due to its repulsive electric field, the interstrip resistance in the device featuring a p-stop implant is high, independently of the applied reverse bias voltage. For the sample without isolation implant, a very low interstrip resistance is observed at low bias voltages. When the bias voltage is increased, the electron layer is removed. Hence, a similar interstrip resistance as for the sensor with a p-stop implant is reached. A conservative estimation of a lower limit for a sufficient interstrip isolation is indicated by the light red line at an interstrip resistance of $R_{\mathrm{int}} \approx 1 \times 10^{8} \Omega \mathrm{cm}$, which is roughly two orders of magnitude above the resistance of the bias resistor $\left(R_{\text {bias }} \approx 2 \mathrm{M} \Omega\right.$ ). This limit will be indicated in all subsequent plots.

The dependence of the interstrip resistance on the bias voltage for the sensor without interstrip implant can be reproduced in the simulation by inserting a fixed charge at the $\mathrm{Si} / \mathrm{SiO}_{2}$ interface. A higher bias potential is required to remove the electron layer below the surface for increasing charge concentrations. Hence, the curve's rise shifts to higher reverse bias voltages if more charge is introduced. In the following, simulated curves are always indicated by lines without points. With an effective fixed charge concentration at the oxide/bulk interface of $N_{\text {ox }}=8 \times 10^{10} \mathrm{~cm}^{-2}$ (orange curve in Figure 3) it is possible to properly model the interstrip resistance rise.

\subsection{Isolation Mechanism and Impact of the Doping Concentration}

Another aspect that influences the interstrip resistance, besides the positive charge in the oxide $N_{\text {ox }}$, is the bulk doping concentration $N_{\text {dop. }}$. For sensors used for this study it is $N_{\text {dop }} \approx 4.5 \times 10^{12} \mathrm{~cm}^{-3}$. The impact on the strip isolation derived from simulations is illustrated in Figure 4. An effective fixed positive interface charge concentration of $N_{\mathrm{ox}}=3 \times 10^{11} \mathrm{~cm}^{-2}$ has been implemented. This is a typical value after irradiation. It can be seen that a higher doping concentration leads to isolated strips for a smaller applied bias potential. 


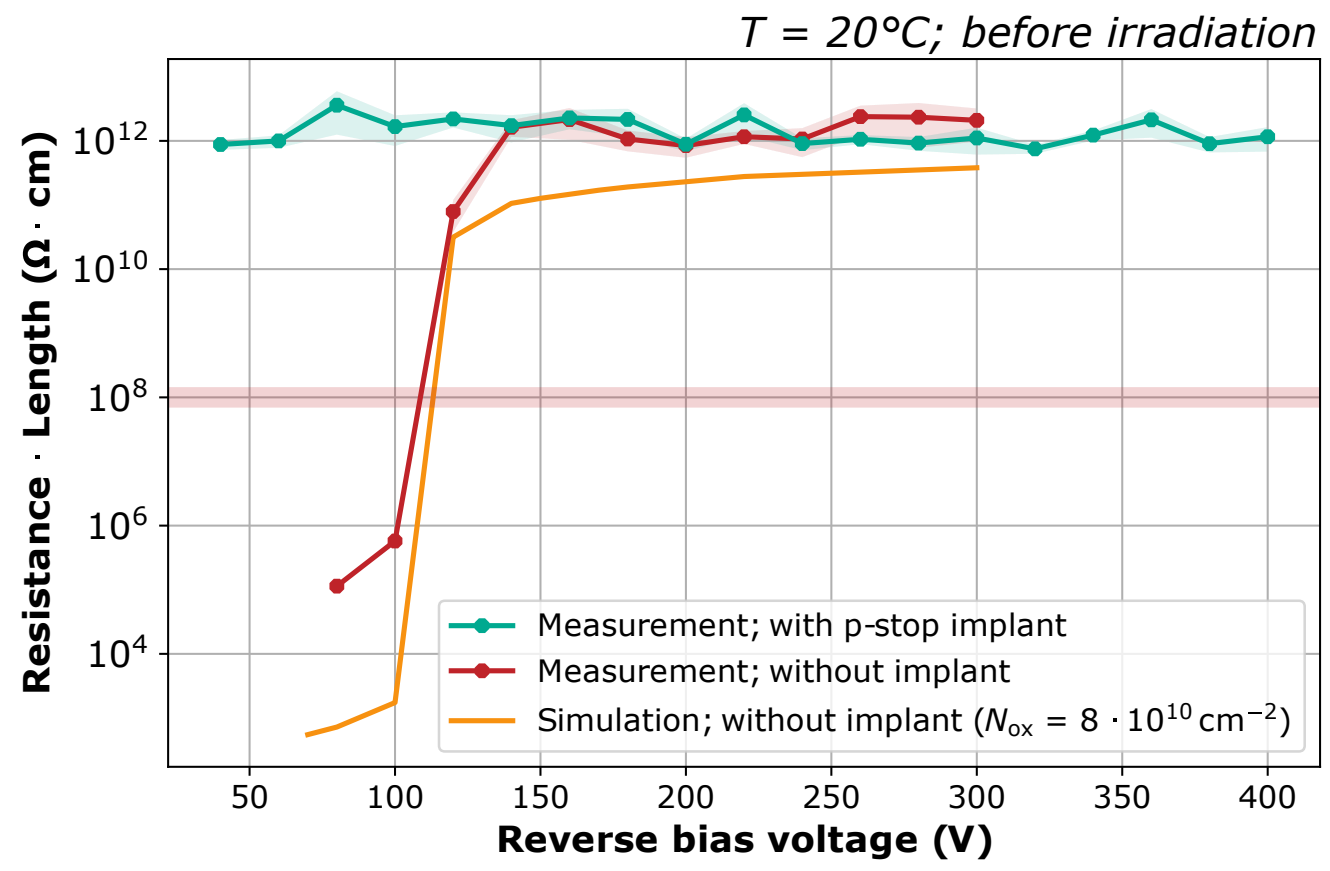

Figure 3: Interstrip resistance in dependence of the reverse bias voltage before irradiation with a conservative estimation for a sufficient value represented by the horizontal red line. A sensor without interstrip isolation implant is indicated in red and another sensor with a p-stop implant in green. The simulated behaviour without isolation implant is represented by the orange curve.

A closer look into the simulated electric field distribution is required in order to understand the impact of the doping concentration. A cut has been applied parallel to the surface $0.5 \mu \mathrm{m}$ below the interface. The $y$ component (perpendicular to the surface) of the electric field is indicated in Figure 5a. Two sensors with different doping concentrations at the same bias voltage of $100 \mathrm{~V}$ are compared. The interstrip resistance is very low in case of $N_{\text {dop }}=5 \times 10^{13} \mathrm{~cm}^{-3}$ and very high for $N_{\text {dop }}=1 \times 10^{14} \mathrm{~cm}^{-3}$ (as visible in Figure 4). It can be seen that the electric field in $y$ direction is more strongly attracting electrons towards the surface in case of the higher doping concentration. This is expected because the depth of the depletion zone is smaller. Hence, the gradient of the potential, the electric field, is higher. When electrons are attracted more strongly towards the surface, the build-up of the electron inversion layer is promoted. This would contradict the outcome that the strip isolation is improved due to higher bulk doping concentrations. In order to understand the ongoing mechanism, the $x$ component of the electric field has to be considered. As illustrated in Figure $5 \mathrm{~b}$ the $x$ component is considerably larger in case of the higher doping concentration. Here, a zoom onto the left half of the device is shown. The $x$-axis ranges from the right side of the strip implant on the left side to the middle between the two strips at $x=0$. It can be seen that a stronger attractive electric field towards the strips in $x$ direction is established due to the increased doping concentration. This non-zero $x$ component of the electric field between the strips is the reason for the removal of the electron layer. The second electric field spike in the figure is caused by the metal overhang. 


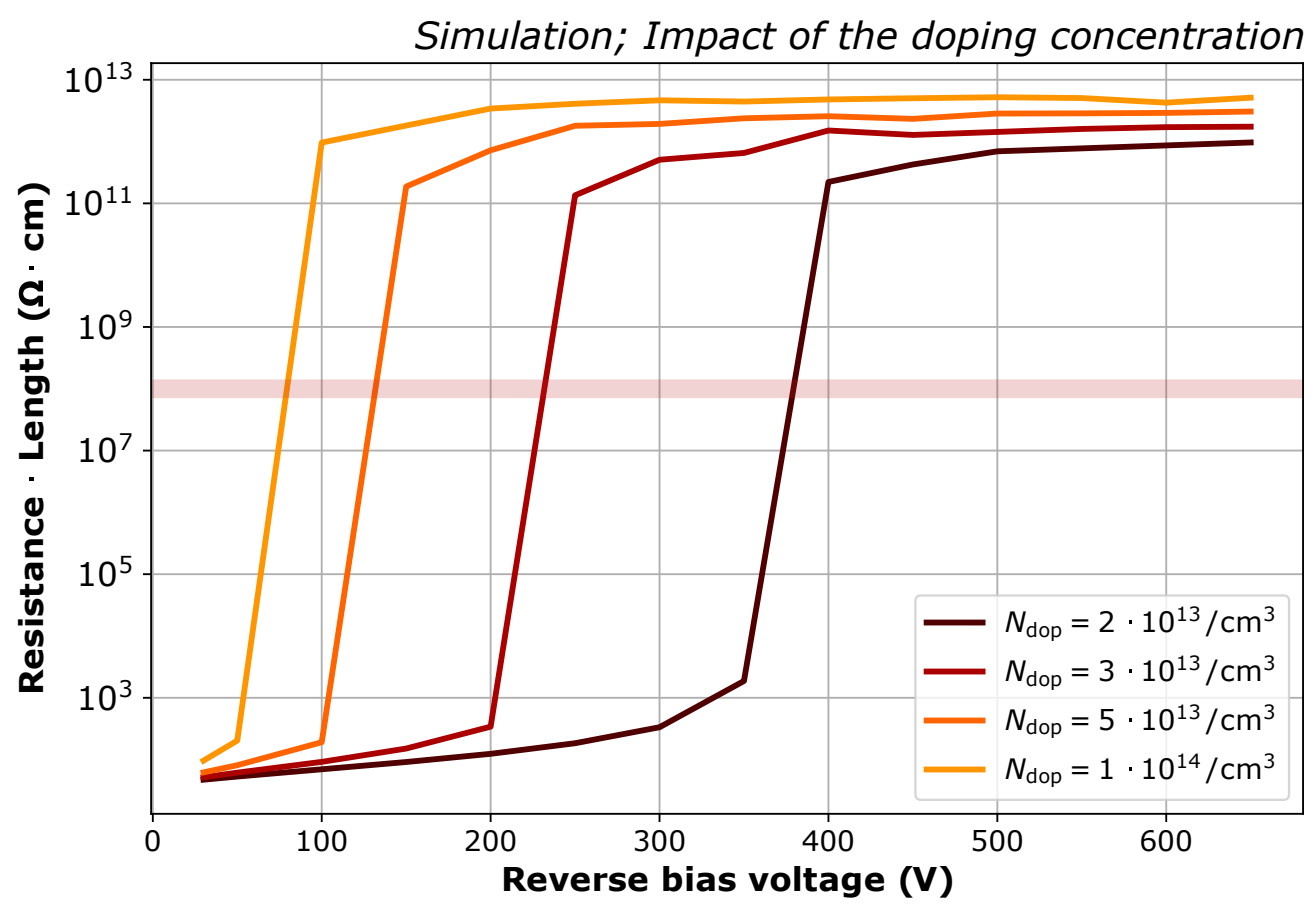

Figure 4: Interstrip resistance in dependence of the reverse bias voltage, for different doping concentrations. A positive charge concentration of $N_{\mathrm{ox}}=3 \times 10^{11} \mathrm{~cm}^{-2}$ is inserted in the oxide/bulk interface.

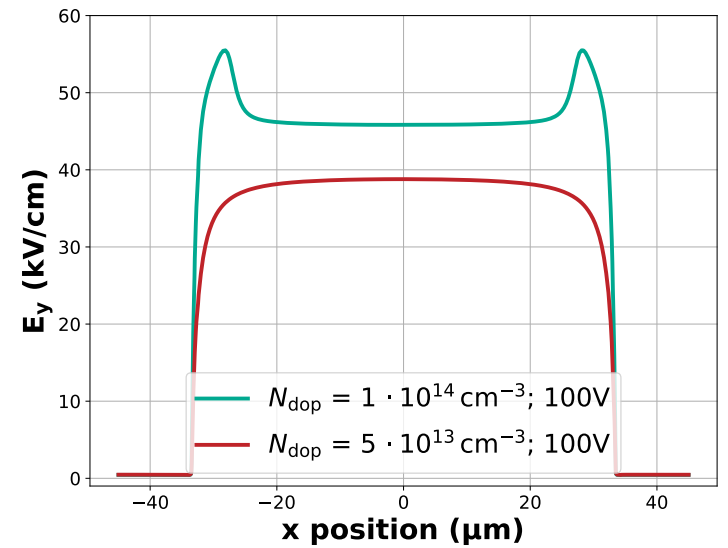

(a)

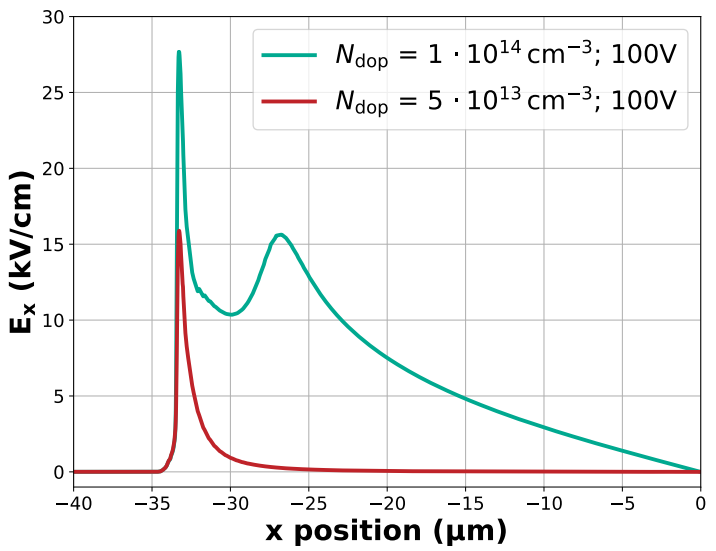

(b)

Figure 5: Electric field's $y$ (a) and $x$ (b) component $0.5 \mu \mathrm{m}$ below the surface for two different doping concentrations at $100 \mathrm{~V}$. The $y$ direction points perpendicular and the $x$ direction parallel to the surface. The position $x=0 \mu \mathrm{m}$ refers to the middle between the two strips and the strip implants start at $x= \pm 34 \mu \mathrm{m}$. A 2D plot of the geometry can be found in Figure 2a. 


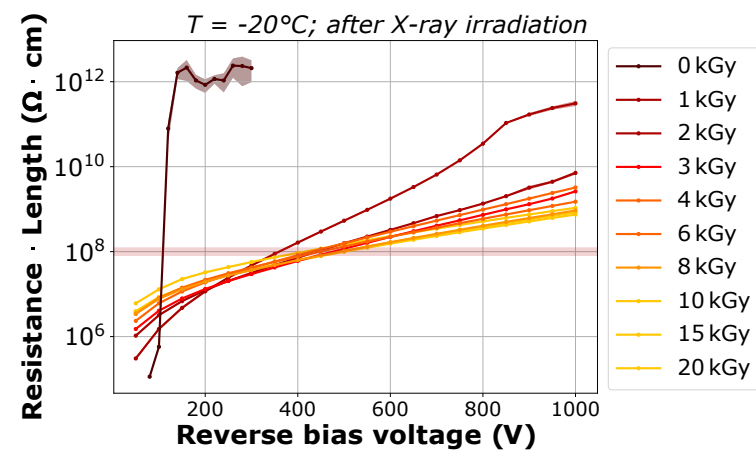

(a)

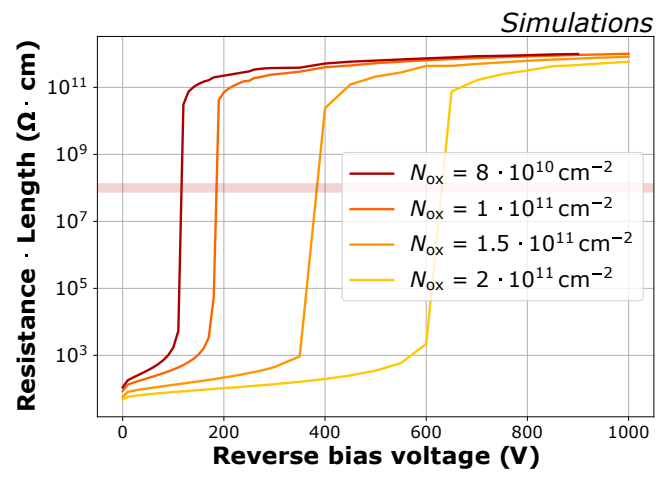

(b)

Figure 6: Interstrip resistance measurements for different doses as a function of the reverse bias voltage (a) and simulated impact of an increase of the positive fixed charge concentration (b).

\section{Interstrip Resistance with pure Surface Damage}

A sensor without isolation implant was irradiated gradually with X-rays until no significant changes of the interstrip resistance could be observed anymore at values of 10 to $20 \mathrm{kGy}$ (applied dose in $\mathrm{SiO}_{2}$ ). Irradiation with $\mathrm{X}$-rays was performed with a tungsten tube at $60 \mathrm{kV}$ and $30 \mathrm{~mA}$ current $[6,7]$. A vanadium filter $(15 \mu \mathrm{m})$ was used to reduce the low energy part of the X-ray spectrum. During irradiation the temperature $T$ has been controlled to $T<-10^{\circ} \mathrm{C}$ in order to prevent annealing and no bias voltage has been applied to the sensor. The measurements were performed at $T=-20^{\circ} \mathrm{C}$ in order to stay consistent with measurements after hadron irradiation. Before irradiation, the measurement temperature did not change the results.

\subsection{Experimental Results}

The outcome of the interstrip resistance measurements for different applied doses is illustrated in Figure 6a. The interstrip resistance is decreasing as expected due to the surface damage induced by the X-ray irradiation, but only for the high bias voltage regime. For low bias voltages the interstrip resistance is increasing with increasing dose, which cannot be explained by a simple damage mechanism which solely introduces positive charge.

\subsection{Surface Defect Simulations}

The impact of an increase of the fixed oxide charge concentration in simulations is illustrated in Figure $6 \mathrm{~b}$. It can be seen that the increase of the effective oxide charge leads to a shift of the whole curve to higher bias voltages. This shows that it is not possible to reproduce the experimental results shown in Figure 6a by exclusively varying the oxide charge concentration. Hence, a more advanced surface defect model is required which includes different types of defects.

In order to augment the defect model, charged traps at the $\mathrm{Si} / \mathrm{SiO}_{2}$ interface (interface traps) are taken into account. The desired effect is that the dynamic occupation of the traps on the one hand counteracts the positive fixed charge for small bias voltages, on the other hand might add to the fixed positive charge at high voltages. This double-edged effect can be achieved by acceptor 
and donor states at the interface. Acceptor states are negatively charged when occupied, thus might partially compensate the positive charge. Contrary, donor states are positively charged when occupied. The general presence of acceptor and donor states at the interface has been evaluated by electron paramagnetic resonance (EPR) measurements [8, 9]. In Ref. [10] it has been shown that it is possible to properly describe a $\mathrm{Si} / \mathrm{SiO}_{2}$ interface with a combination of fixed positive charge and at least three different interface defects after X-ray irradiation.

Because surface effects of devices with similar base material could be described with a TCAD model presented in Ref. [11], a similar model is used for this study. Three different defect states at the $\mathrm{Si} / \mathrm{SiO}_{2}$ interface are introduced in the simulations in order to take interface traps into account. The energy levels of the interface trap states are Gaussian distributed within the silicon bandgap. The trap types with the corresponding peak energies and Gaussian width $(\sigma)$, as suggested in Ref. [11], are given in Table 1. The capture cross sections for electrons as well as for holes are fixed to $1 \times 10^{-15} \mathrm{~cm}^{2}$. An interface trap concentration $N_{\mathrm{it}}\left(\mathrm{cm}^{-2}\right)$ is required as input parameter for the simulation framework determining the number of traps. The same amount of donors and acceptors is introduced proportionally to the trap concentration. Donor states are located around a peak energy of $0.7 \mathrm{eV}$ above the valence band edge $E_{\mathrm{v}}$. Since the model incorporates two acceptor trap types, the introduction rate is shared between them. The acceptor traps with a peak energy of $0.4 \mathrm{eV}$ below the conduction band edge $E_{\mathrm{c}}$ are introduced with $40 \%$ and the states at $0.6 \mathrm{eV}$ below the conduction band edge with $60 \%$ of $N_{\mathrm{it}}$.

Table 1: Interface traps according to the surface defect model described in Ref. [11].

\begin{tabular}{|l|c|c|c|c|}
\hline Trap type & Peak energy $(\mathrm{eV})$ & $\sigma(\mathrm{eV})$ & Capture cross section $\left(\mathrm{cm}^{2}\right)$ & Fraction of $N_{\mathrm{it}}$ \\
\hline Donor & $E_{\mathrm{v}}+0.70$ & 0.07 & $1 \times 10^{-15}$ & $100 \%$ \\
Acceptor & $E_{\mathrm{c}}-0.40$ & 0.07 & $1 \times 10^{-15}$ & $40 \%$ \\
Acceptor & $E_{\mathrm{c}}-0.60$ & 0.07 & $1 \times 10^{-15}$ & $60 \%$ \\
\hline
\end{tabular}

In contrast to Ref. [11], a dose independent fixed ratio between the oxide charge and interface trap concentration is a priori not assumed. Therefore, the oxide charge and interface trap concentrations have to be determined. Both parameters have been adjusted in the simulation so that the measured behaviour of the interstrip resistance is replicated after every step of applied X-ray dose. The "Optimizer" tool of the Synopsys Sentaurus TCAD framework has been utilised to vary the defect concentrations until the resulting interstrip resistance versus bias voltage curve matches the measured curve as closely as possible. An example of such a comparison between measurements and simulations is shown in Figure 7 for a dose of $8 \mathrm{kGy}$. The simulated behaviour is in good agreement with the measured curve (barely visible due to the high match). The other dot-dashed lines indicate the simulation results for defect concentrations slightly deviating from the optimum. A lower $N_{\text {it }}$ as well as a higher $N_{\text {ox }}$ result in less interstrip isolation. However, the impact of $N_{\text {ox }}$ and $N_{\text {it }}$ is not undistinguishable since $N_{\text {it }}$ affects the slope whereas $N_{\text {ox }}$ shifts the whole curve to higher bias voltages. Increasing the interface trap concentration leads to a higher interstrip resistance, as indicated by the green line.

A summary of the obtained positive oxide charge and interface trap concentrations as a function of the radiation dose is provided in Figure 8. The defect concentrations increase with the dose until 


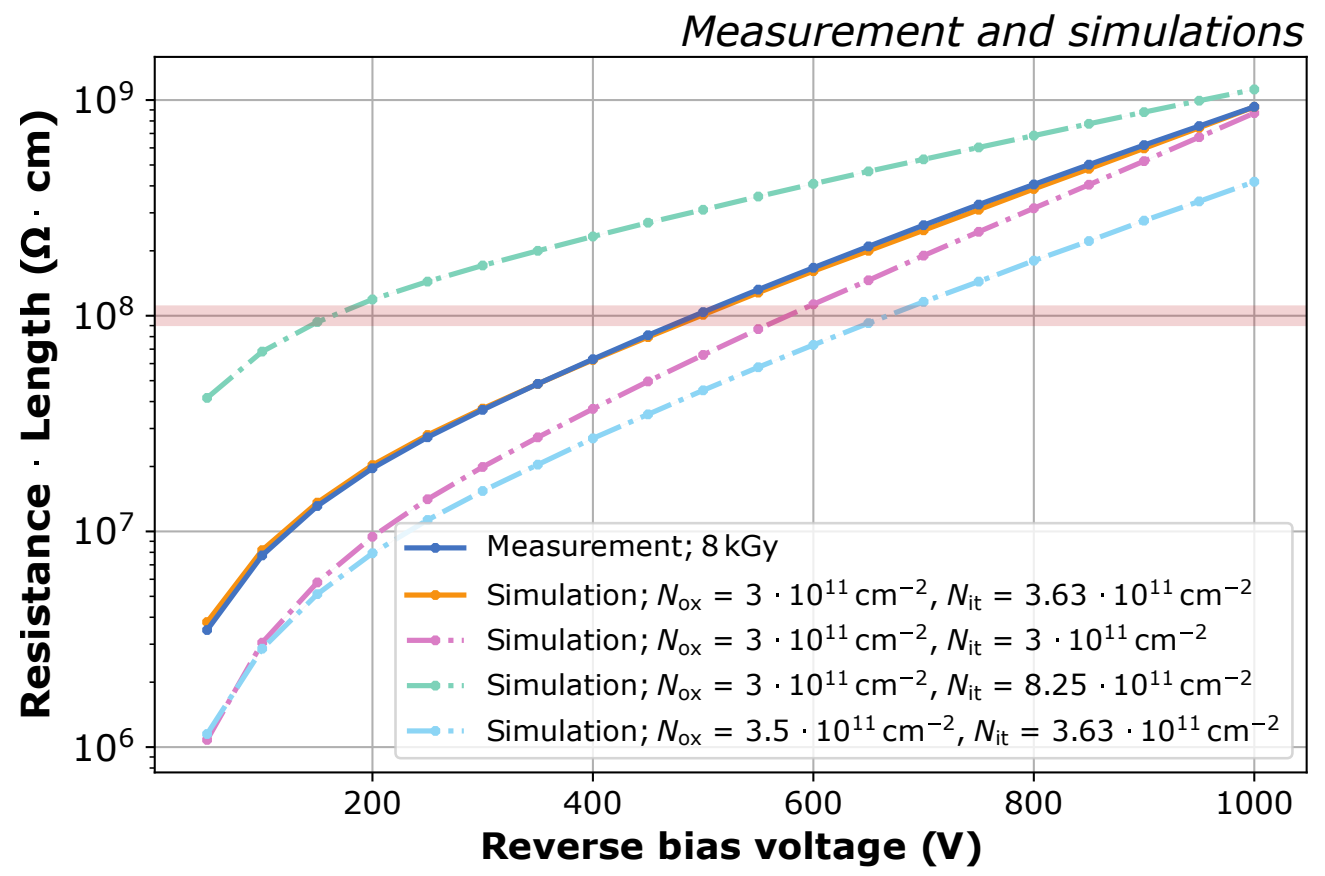

Figure 7: Example of a comparison of the interstrip resistance dependent on the applied bias voltage between measurements (blue with dots) and simulations (optimised curve in orange; other colours with variations of $N_{\mathrm{ox}}$ and $N_{\mathrm{it}}$ to illustrate dependencies) after irradiation to $8 \mathrm{kGy}$.

saturation is reached at 10 to $15 \mathrm{kGy}$, approximately. As shown in the figure, the defect saturation concentrations are $N_{\mathrm{ox}} \approx 3 \times 10^{11} \mathrm{~cm}^{-2}$ and $N_{\mathrm{it}} \approx 4 \times 10^{11} \mathrm{~cm}^{-2}$.

With these investigations the simulation model to describe pure surface damage, applied by $\mathrm{X}$-ray irradiation, is validated. It has been observed that saturation of the simulation parameters $N_{\text {ox }}$ and $N_{\text {it }}$ can be expected above an applied dose of $15 \mathrm{kGy}$ in $\mathrm{SiO}_{2}$. Defect concentrations were extracted depending on the applied dose.

\section{Interplay of Surface and Bulk Defects}

\subsection{Irradiation Procedures}

A set of strip sensors without strip isolation implant has been irradiated with neutrons to fluences of $3 \times 10^{14} \mathrm{n}_{\mathrm{eq}} / \mathrm{cm}^{2}$ and $6 \times 10^{14} \mathrm{n}_{\mathrm{eq}} / \mathrm{cm}^{2}$ and with protons to a fluence of $6 \times 10^{14} \mathrm{n}_{\mathrm{eq}} / \mathrm{cm}^{2}$. For comparison, one sensor with an atoll-shaped p-stop implant $\left(5 \times 10^{15} \mathrm{~cm}^{-3}\right.$ peak doping concentration) has been irradiated with protons to a fluence of $6 \times 10^{14} \mathrm{n}_{\mathrm{eq}} / \mathrm{cm}^{2}$ as well. The irradiation with neutrons was performed inside a spallation reactor in Ljubljana, Slovenia [12]. Due to photon contamination inside the reactor, the estimated additional radiation dose is $1 \mathrm{kGy}$ per $1 \times 10^{14} \mathrm{n}_{\text {eq }} / \mathrm{cm}^{2}$ [13]. The proton irradiation was performed at ZAG (Eggenstein-Leopoldshafen, Germany) with $23 \mathrm{MeV}$ protons, where the samples are homogeneously irradiated in a cold atmosphere $[14,15]$. Contrary to neutrons, protons ionise the material, which leads to an estimated dose of $145 \mathrm{kGy}$ per $1 \times 10^{14} \mathrm{n}_{\mathrm{eq}} / \mathrm{cm}^{2}$ (calculated via Ref. [16]). 


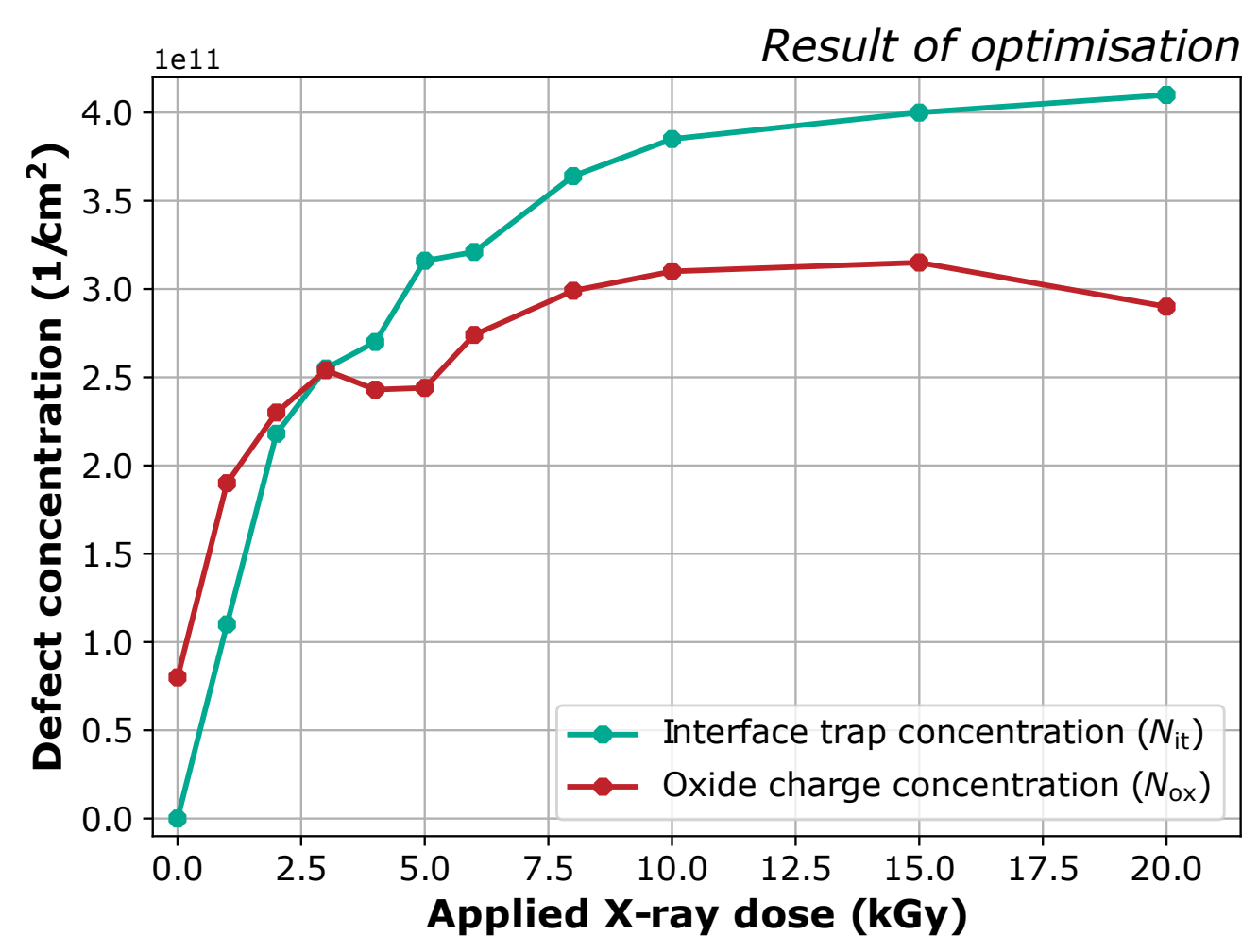

Figure 8: Summary of the defect concentrations dependent on the applied dose. The effect of $\mathrm{X}$-ray irradiation on the interstrip resistance saturates at around $15 \mathrm{kGy}$.

\subsection{Experimental Results}

The interstrip resistance measurements of samples irradiated with hadrons are summarised in Figure 9. Reference measurements on a sensor which has a p-stop implant and on the sensor irradiated with X-rays of Section 4 are shown for comparison. Sensors which were irradiated with hadrons show a sufficient interstrip resistance above a bias voltage of $100 \mathrm{~V}$. Although the induced doses of neutron and proton irradiation are very different in magnitude, the measured curves are quite close to each other and differ significantly from measurements on pure X-ray irradiated samples.

By comparing the two sensor which were irradiated with neutrons in Figure 9, it can be concluded that a higher bulk defect concentration leads to a higher interstrip resistance. This is in contrast to the fact that the applied dose is doubled from $3 \mathrm{kGy}$ to $6 \mathrm{kGy}$, which leads to larger surface defect concentrations (Figure 8) and therefore a decrease of the interstrip resistance would be expected without taking bulk defects into account, at least above $500 \mathrm{~V}$. Comparing the measurements after hadron irradiation to the measurements on the purely X-ray irradiated sensor shows that the bulk defects introduced by the hadron irradiation have a beneficial effect on the interstrip resistance and compensate the degradation due to surface damage. The significance of this effect becomes clearer when the sensor without isolation implant is compared to the sensor with a p-stop implant at the same fluence. Since the interstrip resistance of these two sensors scales similarly with reverse bias over the whole applied voltage range, it can be concluded that the beneficial effect of the bulk defects is predominant and the p-stop structure loses its impact. 
$T=-20^{\circ} \mathrm{C} ;$ Measurements after irradiation

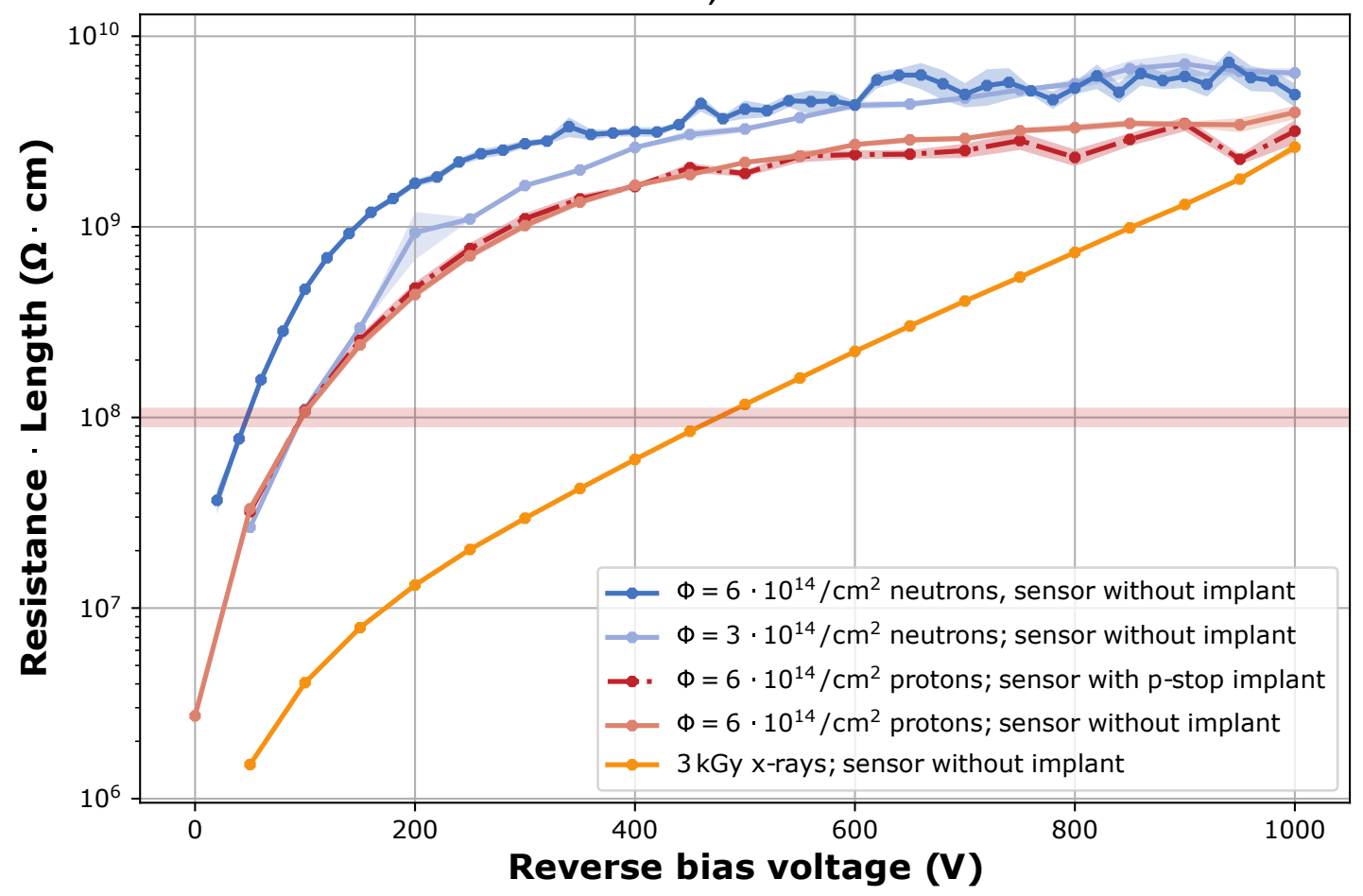

Figure 9: Comparison of the interstrip resistance dependent on the reverse bias voltage for sensors without an isolation implant and one sensor with a p-stop implant (dot-dashed). Neutron irradiation is indicated in blue, proton irradiation in red and X-ray irradiation in orange.

At which fluence the bulk defects become relevant regarding surface properties is of practical relevance. In order to investigate, another set of sensors was irradiated with neutrons to fluences ranging from $1 \times 10^{10} \mathrm{n}_{\mathrm{eq}} / \mathrm{cm}^{2}$ to $1 \times 10^{14} \mathrm{n}_{\mathrm{eq}} / \mathrm{cm}^{2}$. No clear beneficial effect of the bulk defects was observed below $1 \times 10^{14} \mathrm{n}_{\mathrm{eq}} / \mathrm{cm}^{2}$, hence the respective measurements are not shown here. The measurements for a sensor which was irradiated with $1 \times 10^{14} \mathrm{n}_{\mathrm{eq}} / \mathrm{cm}^{2}$, corresponding to an applied dose of $1 \mathrm{kGy}$, is compared in Figure 10 to a sensor which was solely irradiated with $1 \mathrm{kGy} \mathrm{X}$-rays. It can be seen that the interstrip resistance is roughly one order of magnitude higher for the sensor with bulk defects compared to the sensor without bulk defects for voltages below 500V. For higher bias voltages, the interstrip resistance becomes smaller than for the case with pure X-ray irradiation, but the absolute value is still considerably above the conservative estimation of a sufficient interstrip resistance.

\subsection{Simulated Impact of Bulk Defects on the Interstrip Isolation}

Bulk defects play a crucial role regarding properties like leakage current, full depletion voltage and charge collection. Leakage current is mainly generated by defects which are located in the middle of the band gap, so that they promote the excitation for electrons of the valence band. The full depletion voltage is increased by charged acceptor or donor states close to the valence or conduction 


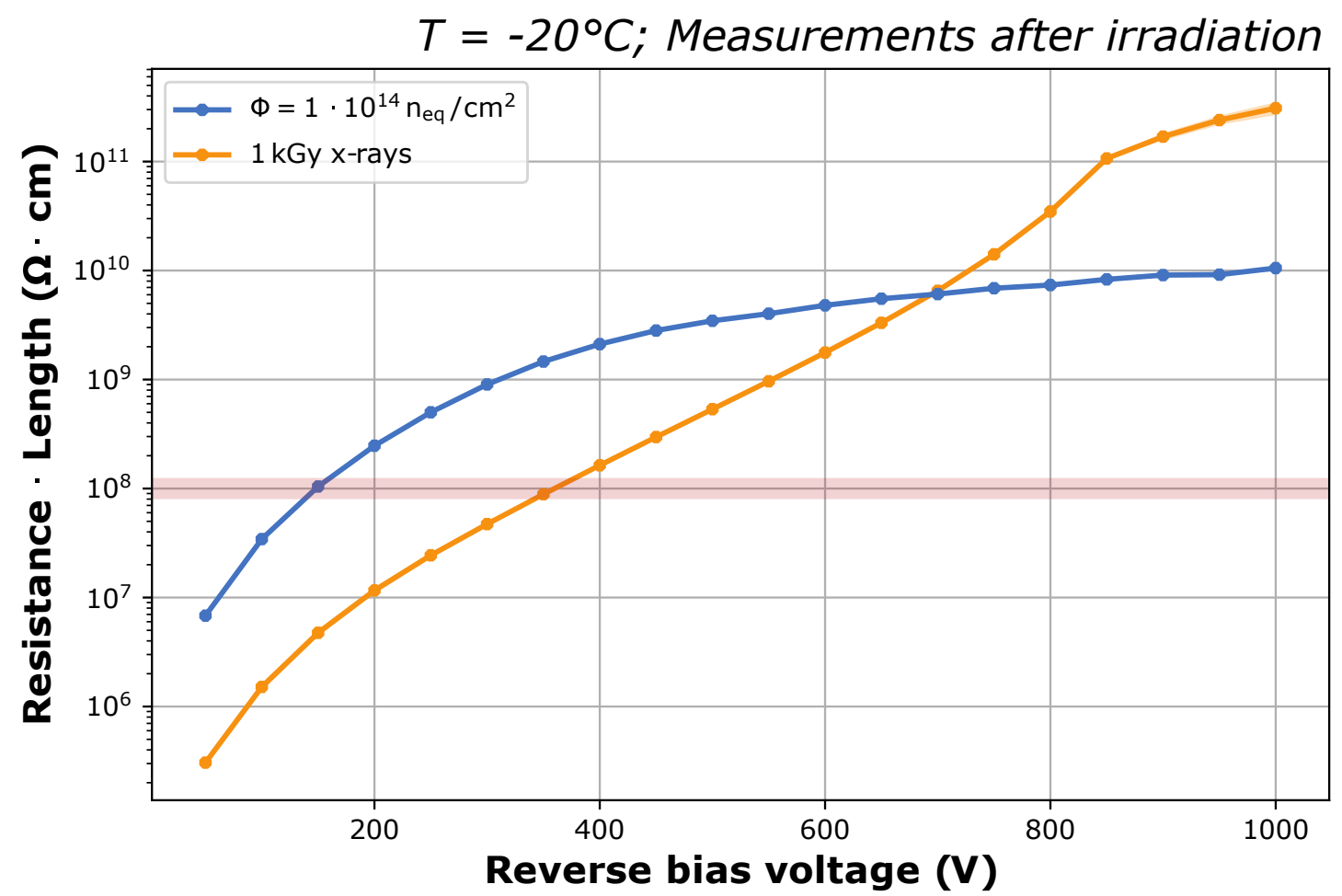

Figure 10: Comparison of the interstrip resistance denpendence on the applied bias voltage after neutron irradiation to a fluence of $1 \times 10^{14} \mathrm{n}_{\mathrm{eq}} / \mathrm{cm}^{2}$ with a corresponding dose of $1 \mathrm{kGy}$ and pure X-ray irradiation to $1 \mathrm{kGy}$.

band edge, respectively [17].

The development of the bulk defect model for TCAD simulations used in this publication is described in Ref. [18]. This defect model is an effective two-trap model and consists of one donor and one acceptor trap type. Details about the defect model for proton irradiation can be found in Table 2 and for neutron irradiation in Table 3. Both models include the donor and acceptor trap at the same energy levels but the introduction rate and the capture cross sections differ. These values have been tuned on diodes and strip sensors such that leakage currents, depletion voltages, electric field distributions (from Transient Current Technique (TCT) measurements) and charge collection measurements are reproduced, as described in the reference.

Table 2: Bulk defect model for sensors irradiated by protons, validated at $T=-20^{\circ} \mathrm{C}$ for fluences ranging from $\Phi=1 \times 10^{14} \mathrm{n}_{\mathrm{eq}} / \mathrm{cm}^{2}$ to $\Phi=1 \times 10^{15} \mathrm{n}_{\mathrm{eq}} / \mathrm{cm}^{2}$ [18]

\begin{tabular}{|l|c|c|c|}
\hline Trap type & Energy level $(\mathrm{eV})$ & Defect concentration $\left(\mathrm{cm}^{-3}\right)$ & Capture cross section $\left(\mathrm{cm}^{2}\right)$ \\
\hline Donor & $E_{\mathrm{v}}+0.48$ & $\Phi \cdot 5.598-3.949 \times 10^{14}$ & $1 \times 10^{-14}$ \\
Acceptor & $E_{\mathrm{c}}-0.525$ & $\Phi \cdot 1.189+6.454 \times 10^{13}$ & $1 \times 10^{-14}$ \\
\hline
\end{tabular}

Before simulations are compared to measurements, it is illustrated how the bulk defects influence the strip isolation. As an example, the impact of proton induced bulk defects according to 
Table 3: Bulk defect model for sensor irradiated by neutrons, validated at $T=-20^{\circ} \mathrm{C}$ for fluences ranging from $\Phi=1 \times 10^{14} \mathrm{n}_{\mathrm{eq}} / \mathrm{cm}^{2}$ to $\Phi=1 \times 10^{15} \mathrm{n}_{\mathrm{eq}} / \mathrm{cm}^{2}$ [18]

\begin{tabular}{|l|c|c|c|}
\hline Trap type & Energy level $(\mathrm{eV})$ & Defect concentration $\left(\mathrm{cm}^{-3}\right)$ & Capture cross section $\left(\mathrm{cm}^{2}\right)$ \\
\hline Donor & $E_{\mathrm{v}}+0.48$ & $\Phi \cdot 1.395$ & $1.2 \times 10^{-14}$ \\
Acceptor & $E_{\mathrm{c}}-0.525$ & $\Phi \cdot 1.55$ & $1.2 \times 10^{-14}$ \\
\hline
\end{tabular}

Table 2 is studied in simulations by increasing the fluence, while the fixed positive charge concentration is kept constant at a value of $3 \times 10^{11} \mathrm{~cm}^{-2}$. Since this investigation aims to gain a better understanding of the impact of bulk defects, interface traps are excluded for now in order to simplify the result. The impact of an increasing fluence on the interstrip resistance is illustrated in Figure 11. It can be seen that the bulk defects effectively shift the sharp increase of the curve to lower bias voltages while the shape of the curve stays the same.

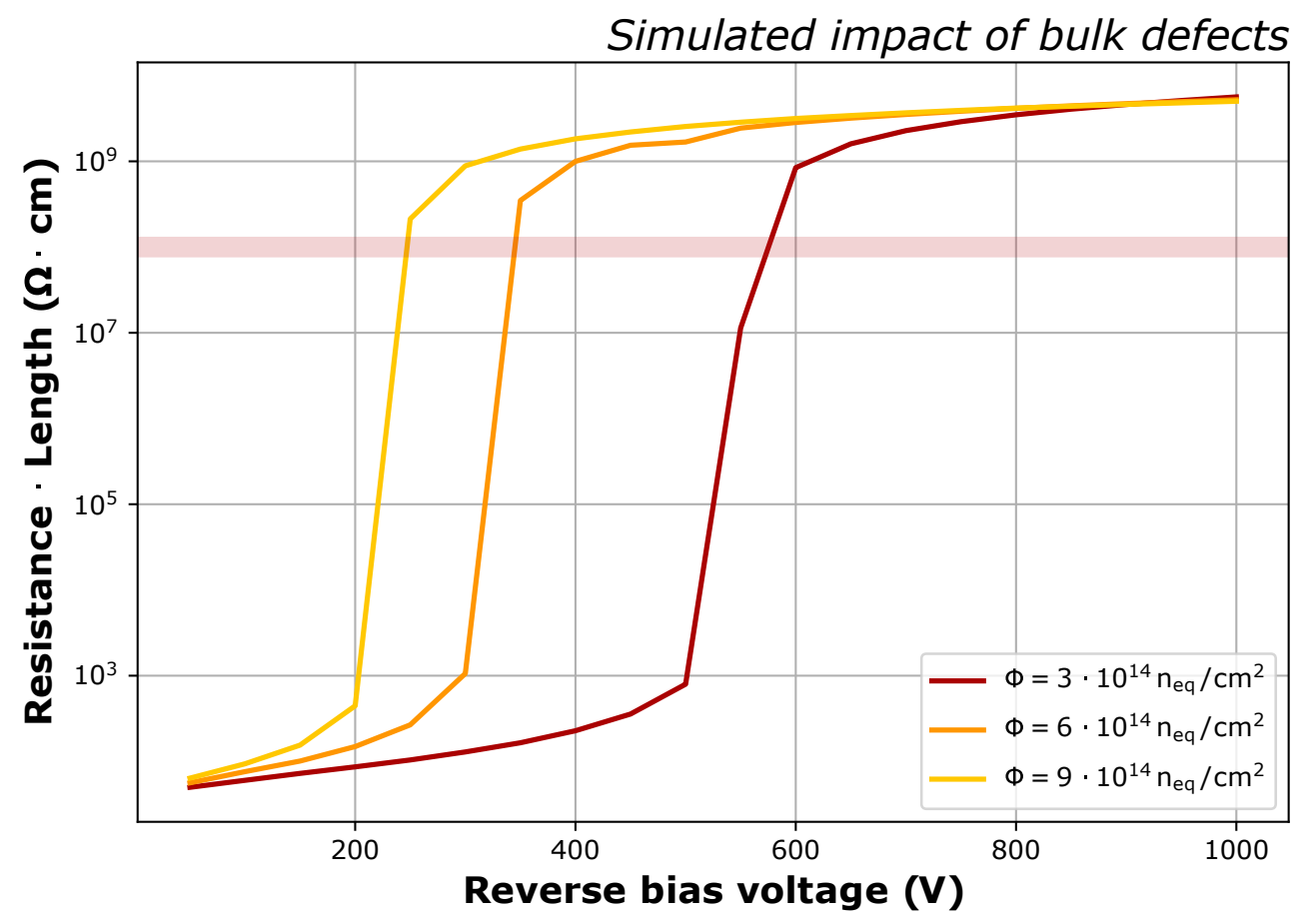

Figure 11: Simulated interstrip resistance dependent on the applied bias voltage. The simulation includes positive fixed charge and bulk defects corresponding to proton irradiation. Brighter colours correspond to higher fluences. The fluence dependence shows the beneficial effect of bulk defects.

Similar to the investigations in Section 3.2 a closer look into the near-surface electric field distribution is required. For this, the two bulk defect concentrations according to the fluences of $6 \times 10^{14} \mathrm{n}_{\mathrm{eq}} / \mathrm{cm}^{2}$ and $9 \times 10^{14} \mathrm{n}_{\mathrm{eq}} / \mathrm{cm}^{2}$ are considered. Figure 11 shows that at $300 \mathrm{~V}$ the lower fluence results in a poor and the higher fluence in a sufficient strip isolation. A cut is applied in the 2D plot of the electric field parallel to the oxide/bulk interface $0.5 \mu \mathrm{m}$ deep in the bulk, similar to the approach in Section 3.2. Along this line the $y$ component, perpendicular to the surface, for the two fluences at $300 \mathrm{~V}$ is shown in Figure 12a. It can be seen that the electric field attracts electrons 


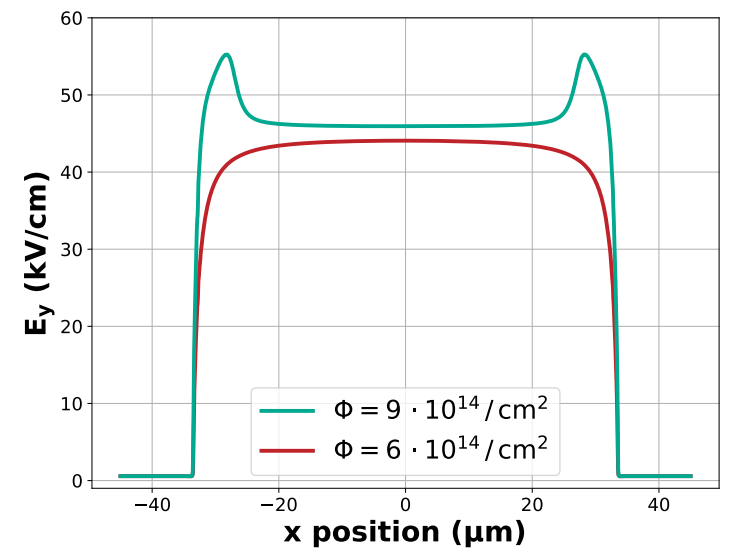

(a)

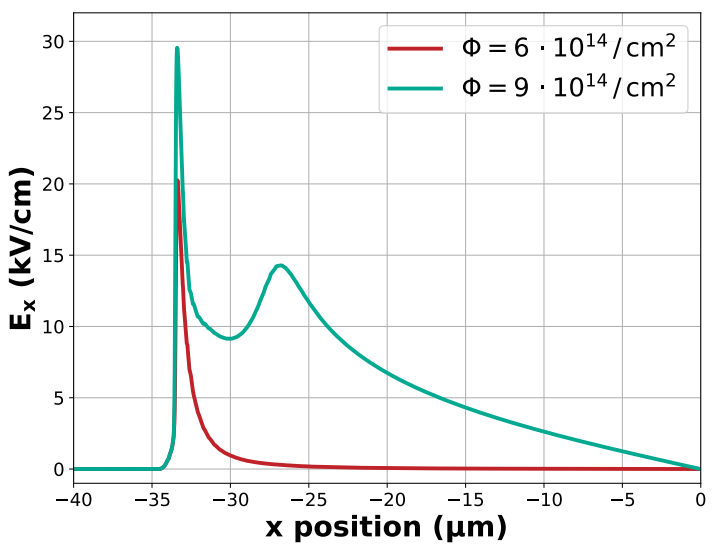

(b)

Figure 12: Electric field's $y$ (a) and $x$ (b) component $0.5 \mu \mathrm{m}$ below the surface for two different fluences at $300 \mathrm{~V}$. The $y$ direction points perpendicular and the $x$ direction parallel to the surface. The position $x=0 \mu \mathrm{m}$ refers to the middle between the two strips and the strip implants start at $x= \pm 34 \mu \mathrm{m}$. A 2D plot of the geometry can be found in Figure 2a.

stronger towards the surface in case of the higher fluence which would contradict the observation of well isolated strips. However, by taking into account the $x$ component of the electric field, which is illustrated in Figure 12b, it can be seen that the electrons are attracted strongly towards the strips. This inhibits the build-up of a homogeneous electron layer. As explained in Section 3.2 the second peak at $x=-27.5 \mu \mathrm{m}$ is caused by the metal overhang. It can be concluded that the increase of the effective doping concentration by hadron irradiation leads to electric fields, pointing towards the strips in $x$ direction, and thus help the strip isolation.

In contrast to the case of a certain doping concentration pre irradiation, the effective doping concentration due to hadron irradiation increases on the basis of defect states distributed over the band gap. Therefore, these bulk defects act additionally as generators of free charges thus increasing the leakage current. Hence, the bulk defects would establish a lower limit of the interstrip resistance in case other isolating measures were not effective anymore, but decrease the maximum achievable interstrip resistance by the continuous generation of free charges. This results in the situation that at high bias voltages, where a sufficient strip isolation is reached, the maximum interstrip resistance is lower compared to the situation with pure fixed charge (Figure 6b). It also explains why the interstrip resistance in Figure 10 of the neutron irradiated sensor stays below the purely X-ray irradiated sensor at high bias voltages.

\subsection{Comparison of Simulations to Experimental Results}

An effective model of defects in the interface which describes pure surface related damage in the silicon oxide and the interface has been established in Section 4. Additionally, a relation between the defect concentrations (effective positive oxide charge $N_{\text {ox }}$ and interface traps $N_{\text {it }}$ ) and the applied dose has been obtained. This can be used to simulate the behaviour of the interstrip isolation when additional bulk defects are introduced. A comparison between the measurements of the samples irradiated with neutrons and the respective simulations is shown in Figure 13a. The measurements 


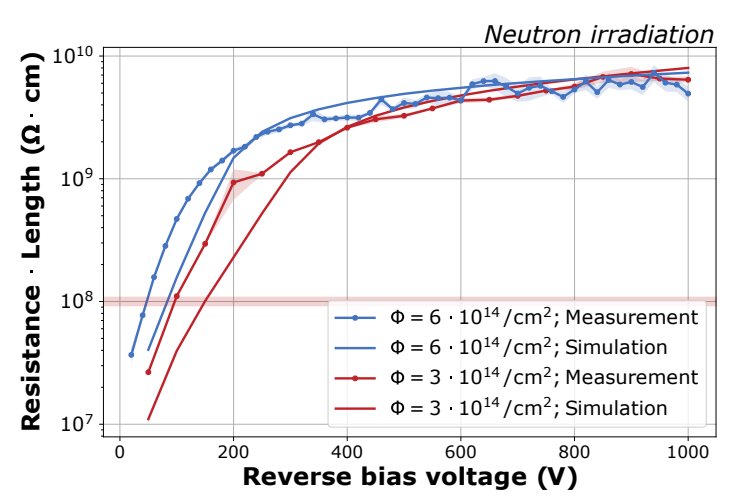

(a)

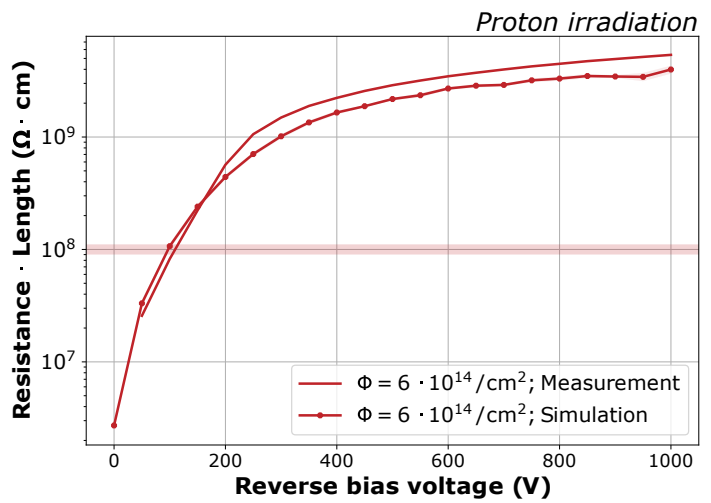

(b)

Figure 13: Comparison of measurements of the interstrip resistance dependent on the reverse bias voltage with simulations for neutron irradiation at different fluences (a) and for proton irradiation at a fluence of $6 \times 10^{14} \mathrm{n}_{\mathrm{eq}} / \mathrm{cm}^{2}(\mathrm{~b})$.

were already shown in Figure 9. These simulations are obtained by introducing the bulk defects corresponding to the fluence and the interface defects corresponding to the findings from Section 4 at the respective dose. The estimated applied dose for $3 \times 10^{14} \mathrm{n}_{\mathrm{eq}} / \mathrm{cm}^{2}$ and $6 \times 10^{14} \mathrm{n}_{\mathrm{eq}} / \mathrm{cm}^{2}$ is $3 \mathrm{kGy}$ and $6 \mathrm{kGy}$, respectively. Figure 13a demonstrates the very good agreement of measured and simulated interstrip resistance curves, which validates the application of the combined surface and bulk model.

With the same procedure it is possible to replicate the interstrip resistance curve of the proton irradiated sample. In this case the fluence is $6 \times 10^{14} \mathrm{n}_{\mathrm{eq}} / \mathrm{cm}^{2}$, which corresponds to an applied dose of $870 \mathrm{kGy}$. Since at this high dose the surface defect concentration is in saturation (Section 4), the respective interface defect concentration in saturation (at $15 \mathrm{kGy}$ ) is used for the simulation. The comparison of the measurements and the simulations is shown in Figure 13b. Again, the measurement is modelled accurately.

The two neutron irradiated sensors were further irradiated with X-rays in order to systematically induce additional surface defects. The resulting decreased interstrip resistance is shown in Figure 14a. However, the interstrip resistance is still significantly higher than in the case of pure $\mathrm{X}$-ray irradiation without neutrons. This shows once more that the bulk defects play a crucial role and preserve the strip isolation. The X-ray irradiation dose was set to $20 \mathrm{kGy}$, which is well in saturation. The samples were also irradiated to higher doses where no difference in the curve could be observed anymore. Hence, for the sake of visibility, these curves are not shown here. Since the surface defects are saturated, the corresponding saturation concentrations of the fixed positive charge and the interface traps derived from the previous X-ray study are used. As can be seen from the Figure 14a, the curve is well reproduced for lower bias voltages and interstrip resistances but overestimates the interstrip resistance for higher values. Our conclusion is that the saturation interface defect concentrations might be higher or different than for pure X-ray irradiation. These higher saturation concentrations could be caused by an unknown effect by which neutrons generate additional states in the oxide. In order to investigate such a possible effect further, another sensor 


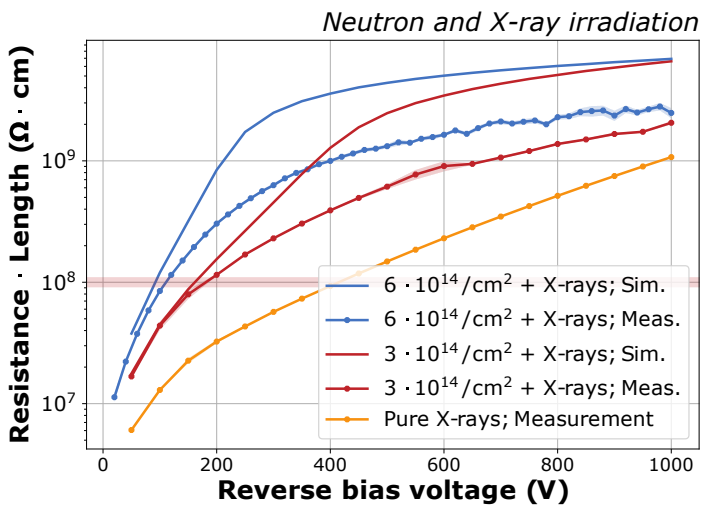

(a)

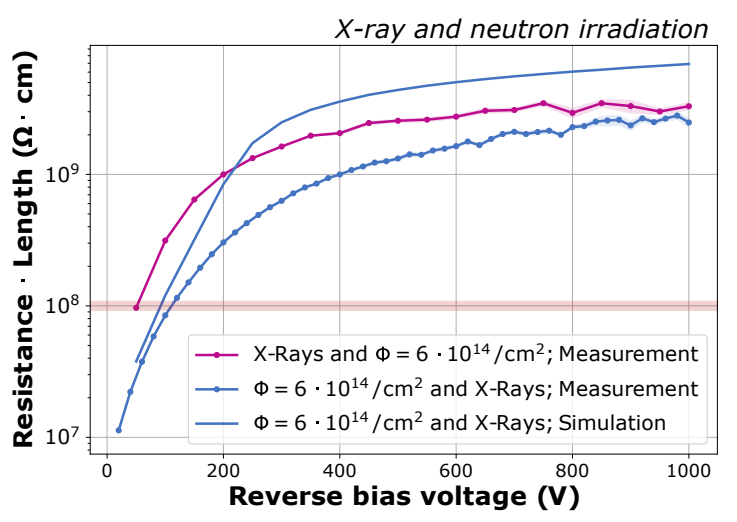

(b)

Figure 14: Comparison of measurements and simulations of the interstrip resistance dependent on the reverse bias voltage for neutron irradiation, followed by $20 \mathrm{kGy}$ X-ray irradiation (a). The orange curve refers to pure X-ray irradiation to $20 \mathrm{kGy}$. Comparison to the reverse sequence, i.e. $\mathrm{X}$-ray irradiation to $20 \mathrm{kGy}$ followed by neutron irradiation to a fluence of $6 \times 10^{14} \mathrm{n}_{\mathrm{eq}} / \mathrm{cm}^{2}$ (b).

was irradiated in reversed order, with X-rays first and $6 \times 10^{14} \mathrm{n}_{\mathrm{eq}} / \mathrm{cm}^{2}$ neutrons afterwards. A difference between the two irradiation sequences was indeed observed, as indicated in Figure 14b. The measurement of the sensor which was irradiated with X-rays first and afterwards with neutrons shows a considerably higher interstrip resistance compared to the other sensor, especially at lower voltages. The reason why this dependence of the irradiation order occurs is currently under investigation. There could be defect states which are introduced due to the neutron irradiation which affect the following defect generation due to X-ray irradiation. Currently, only defect generation in the bulk is considered according to the neutron flux in the simulations. However, the effect might also be explained by an unintended annealing during shipment of the samples. The effect of annealing on the interstrip resistance when bulk and surface defects are present at the same time is not trivial.

\section{Conclusion}

A comprehensive irradiation study with n-on-p silicon strip sensors without any interstrip isolation implants has been performed to evaluate the interplay of surface and bulk damage. Estimations of the interstrip isolation of sensors for tracking detectors at high luminosity collider experiments based on simulations are usually done on the basis of pure ionising radiation. By comparing interstrip resistance measurements on sensors after irradiation with different particle compositions, especially hadrons, to TCAD simulations, this study shows that it is not possible to get an accurate prediction of the interstrip isolation with this approach. Instead, for fluences above $1 \times 10^{14} \mathrm{n}_{\mathrm{eq}} / \mathrm{cm}^{2}$ it is necessary to consider the bulk defects as well in order to get a comprehensive description of the interstrip isolation. A compound model is suggested, which consists of an effective positive fixed charge, three interface traps (following Ref. [11]) together with two bulk defects (following Ref. [18]). With this model it was possible to describe the measurements after irradiation, especially with protons and neutrons where bulk and surface damage occur at the same time. 
The results also put previous investigations concerning the impact of a p-stop or p-spray isolation implant into perspective. In the past, it has been claimed that such implants preserve the interstrip isolation in harsh radiation environments, where bulk defects and surface damage is generated. The outcome that the interstrip isolation is preserved can be confirmed by the results of this publication. However, at these high fluences the isolation is preserved due to the crucial impact of the bulk defects on the electric field distribution at the surface and not due to the p-type implant between the strips. The p-stop implant might indeed be required without irradiation or at fluences below $1 \times 10^{14} \mathrm{n}_{\mathrm{eq}} / \mathrm{cm}^{2}$, depending on the oxide quality and doping concentration.

\section{Acknowledgments}

This project has received funding from the European Union's Horizon 2020 Research and Innovation programme under Grant Agreement no. 654168. We would like to thank the Tracker Group of the CMS Collaboration for providing the samples for this study. The authors were partially financially supported by the Federal Ministry of Education and Research of Germany (BMBF) under Grant 05H19VKCC9.

\section{References}

[1] Frank Hartmann, Evolution of silicon sensor technology in particle physics; 2nd ed., Springer, Nov. 3, 2017, DOI: 10.1007/978-3-319-64436-3.

[2] Y. Unno et al., p-Bulk silicon microstrip sensors and irradiation, Nucl. Instrum. Meth. A 579 (2007) 614-622.

[3] CMS Collaboration, The Phase-2 Upgrade of the CMS Tracker - Technical Design Report, CERN-LHCC-2017-009, CMS-TDR-014 (2017).

[4] Jan-Ole Müller-Gosewisch, Irradiation Studies of Charge Collection and Interstrip Resistance of Silicon Strip Sensors in the Course of the CMS Phase-2 Upgrade, 2018, Master Thesis (ETP-KA/2018-21), Karlsruhe Institute of Technology, https://publish.etp.kit.edu/record/21516.

[5] Synopsys Sentaurus, https://www.synopsys.com/silicon/tcad/framework.html, Version 2017.09.

[6] KIT X-Ray Tube, www.etp.kit.edu/english/265.php, accessed on 2020/09/20.

[7] M. Guthoff et al., Geant4 simulation of a filtered X-ray source for radiation damage studies, Nucl. Instrum. Meth. A 675 (2012) 118-122.

[8] C. R. Helms and E. H. Pointdexter, The silicon-silicon dioxide system: Its microstructure and imperfections, Rep. Prog. Phys. 57 (1994) 791-852.

[9] P. Lenahan, Deep level defects involved in MOS device instabilities, Microelectronics Reliability 47 (2007) 890-898.

[10] J. Zhang et al., Study of radiation damage induced by $12 \mathrm{keV}$ X-rays in MOS structures built on high-resistivity n-type silicon, Journal of Synchrotron Radiation 19 (2012) 340-346.

[11] F. Moscatelli et al., Effects of Interface Donor Trap States on Isolation Properties of Detectors Operating at High-Luminosity LHC, IEEE Transactions on Nuclear Science 64 (2017) 2259-2267.

[12] L. Snoj, G. Žerovnik and A. Trkov, Computational analysis of irradiation facilities at the TRIGA reactor, Applied Radiation and Isotopes 70 (2012) 483-488. 
[13] I. Mandić et al., Bulk damage in DMILL npn bipolar transistors caused by thermal neutrons versus protons and fast neutrons, IEEE Transactions on Nuclear Science 51 (2004) 1752-1758.

[14] A. Fugeri, Quality Assurance and Irradiation studies on CMS silicon strip sensors, 2006, $\mathrm{PhD}$ Thesis (IEKP-KA/2005-01), Karlsruhe Institute of Technology, https://publish.etp.kit.edu/record/21628.

[15] Proton Irradiation Facility, www.etp.kit.edu/english/264.php, accessed on 2020/09/20.

[16] NIST, stopping-power and range tables of protons, http://physics.nist.gov/PhysRefData/Star/Text/PSTAR.html, accessed on 2020/09/20.

[17] M. Moll, Radiation damage in silicon particle detectors: Microscopic defects and macroscopic properties, 1999, PhD Thesis (DESY-THESIS-1999-040), Hamburg University, http://cds.cern.ch/record/425274.

[18] Robert Eber, Investigations of new Sensor Designs and Development of an effective Radiation Damage Model for the Simulation of highly irradiated Silicon Particle Detectors, 2013, PhD Thesis (IEKP-KA/2013-27), Karlsruhe Institute of Technology, https://publish.etp.kit.edu/record/20930. 